organisations enhanced bereavement care for families. Links between organisations were achieved through joint learning opportunities and hospice visits.

\section{0-9 HOW DO UK HOSPICES SUPPORT FAMILIES WITH DEPENDENT CHILDREN? RESULTS FROM A NATIONWIDE SURVEY}

${ }^{1}$ Liz Reed, ${ }^{2}$ Jane Cockle-Hearne, ${ }^{1}$ Jennifer Todd, ${ }^{2}$ Emma Ream. ' Princess Alice Hospice, Esher, UK; ${ }^{2}$ University of Surrey, Guildford, UK

\subsection{6/bmjspcare-2018-hospiceabs.9}

Background In the UK, 23000 parents die annually leaving over 40,000 dependent children (Childhood Bereavement Network, 2015). Without support, the impact of parental loss on the social, emotional and physical development of children can last a lifetime (Moore \& Raunch, 2010; Ellis, Dowrick, Lloyd-Williams, 2013). Children value honest communication about parental illness (Raveis, Siegel, Karus, 1999; Christ, Raveis, Siegel et al., 2005), but many parents are unprepared for these conversations and would like more support (Visser, Huizinga, Hoekstra et al., 2006; Semple \& McCance, 2010). UK hospices contribute considerably to the end of life care of more than 200,000 patients a year (Hospice UK), of which anecdotal evidence suggests between $5 \%-10 \%$ have children under 18 years. To date, little data exists on the contribution UK hospices make to supporting families with dependent children.

Aim To examine the support provided by UK hospices to families with dependent children under 18 years when one parent is dying.

Method 197 UK hospices were invited to complete an online survey in March/April 2018. For pre-bereavement and bereavement, the survey asked about support delivered in terms of format, location and recipients and about processes for gathering information and for supporting staff. Region and size of hospice were collected. Responses were anonymous.

Results Response rate was 66\% (130/197). All UK regions were represented. Most hospices provided in-patient and community services and were medium-sized in terms of beds and referrals. Among hospices responding, 78.4\% (87/111) asked about dependent children; a substantial minority had no consistent or formal processes $(21 \% ; 24 / 111)$, and did not provide support for staff $(21.5 \% ; 20 / 93)$. For pre-bereavement and bereavement, written information, signposting to other services and one-to-one support were most delivered formats. Support was least likely to be provided in absence of the dying person.

Conclusion The research provides a comprehensive and representative account of support provided by hospices to families with dependent children prior to, and following, parental death. It identifies gaps in provision and can contribute to developing interventions that can be delivered by UK hospice staff.

\section{0-10 CHILDREN'S PALLIATIVE CARE NURSE ROTATION POSTS: A RECRUITMENT, RETENTION AND RESILIENCE SOLUTION?}

${ }^{1}$ Katie Stevens, ${ }^{1}$ Rachel Black, ${ }^{2} T r i c i a$ Wilcocks. ${ }^{1}$ Children's Hospices Across London, London, UK; ${ }^{2}$ ellenor, Gravesend, UK

10.1136/bmjspcare-2018-hospiceabs.10
Background The National Health Service is experiencing an unprecedented nursing shortfall. For the first time in 2017 more nurses left the profession than joined (Nursing \& Midwifery Council, 2017). Within London competition for children's nurses is strong, with charitable, statutory and private organisations attracting registered staff. The need for children's palliative care (CPC) services is growing within the UK (Together for Short Lives, 2018): however, Dickson (2017) identified an $11 \%$ nursing vacancy rate here with $65 \%$ of these unfilled for $3+$ months. Innovative opportunities for staff development are presented as a tool to aid recruitment and retention (NHS Employers, 2015; Smith \& Baltruks, 2015; The Association of UK University Hospitals, 2017).

This project adopts an iterative approach to design, implementation and review of rotation posts for CPC nurses and will measure the impact on the workforce.

Aim To create and test rotation posts for CPC nurses, subsequently enabling recruitment, development and retention of a resilient CPC nursing workforce, responsive to patient need.

Methods Initial grant application, stakeholder engagement, recruitment of project manager (PM). Literature review completed, hospice nurse survey and hospice retention data gathered, service user involvement, pilot rotation pathways with bespoke competencies developed. Pilot rotations commence September 2018. University partners to develop resilience tool and gather information on programme impact. Clinical supervision and action learning sets integral throughout.

Results Successful Burdett Trust funding bid. Appointment of PM in first recruitment drive. Cumulative enthusiasm established through wide networking. Success is reliant on flexibility and innovation. Clinical competencies gathered, scope for Pan London CPC document development. CPC nurse survey: eagerness amongst staff for new development pathways, especially senior staff. Requesting networking opportunities, good support identified as essential. Literature review: minimal evidence of which interventions influence recruitment and retention, less for hospice workforce. Retention data: shared challenges, improved reporting identified to inform workforce development.

Conclusion Collaborative working, harnessing sector wide enthusiasm, facilitates development of nurse rotation programmes.

\section{Parallel session 4: Innovating and evaluating palliative care}

\section{0-11 THE HOSPICE ENABLED DEMENTIA PARTNERSHIP (HEDP): OVERVIEW OF EVALUATION FROM THREE YEAR PILOT PROJECT}

Joanne Ballentine. Northern Ireland Hospice, Belfast, Antrim

10.1136/bmjspcare-2018-hospiceabs.11

Background Currently over 1400 deaths a year in Northern Ireland, directly attributable to dementia yet, to date, dementia is rarely considered within a palliative care context, with limited attention directed towards the person's psychological, social, emotional and end of life needs or the support of their family carers. To address this need, Northern Ireland Hospice developed an innovative service (HEDP), based on collaborative working across voluntary and statutory 